\title{
Data-Driven Impedance Matching in Multilateral Teleoperation Systems
}

\author{
Mohd Syakirin Ramli ${ }^{1 *}$, Hamzah Ahmad ${ }^{2}$ \\ ${ }^{1}$ Instrumentation and Control Engineering (ICE) Research Group \\ ${ }^{2}$ Faculty of Electrical \& Electronics Engineering, Universiti Malaysia Pahang
}

\section{Article Info \\ Article history: \\ Received Nov 12, 2017 \\ Revised Jan 10, 2018 \\ Accepted Feb 13, 2018 \\ Keywords: \\ Bilateral teleoperation \\ FRIT \\ Impedance matching \\ Laguerre function \\ Least-square method \\ Multi-agents}

\begin{abstract}
This paper addresses a tuning approach to improve transparency between master and slave manipulators in a multilateral teleoperation system. The slave manipulators comprised of multi-agents of simple mass are converted into a bilateral system through the passive decomposition technique. There are two main operations being considered, namely; grasping and handling. The object grasping is achieved through consensus control. For handling, an equalizer constructed by a Laguerre function connected in-feedback-loop to the master manipulator is chosen and tuned to achieve impedance matching between both sides of teleoperation. Fictitious Reference Iterative Tuning (FRIT) is adopted to properly tune the selected equalizer. The result shows that by introducing an optimally tuned equalizer, the overall impedance matching and transparency between the single master and multi-slave manipulators are improved. Finally, the effectiveness of the proposed tuning algorithm is presented through a numerical example.
\end{abstract}

Copyright () 2018 Institute of Advanced Engineering and Science. All rights reserved.

\section{Corresponding Author:}

Mohd Syakirin Ramli,

Instrumentation and Control Engineering (ICE) Research Group,

Faculty of Electrical \& Electronics Engineering,

Universiti Malaysia Pahang,

26600 Pekan, Pahang, Malaysia.

Email: syakirin@ump.edu.my

\section{INTRODUCTION}

Teleoperation systems extend the human operators' capability to perform task at the remote site. In general, the teleoperation systems can be categorized into bilateral teleoperation[1] and multilateral teleoperation[2], [3]. To properly control the multilateral teleoperation system pose some challenges, especially when we consider a single master with multi-slaves configuration. Generally, there is inadequate degree-of-control on the master side to effectively manipulate the slave side, hence requires the slaves to have a certain degree of self-control. To address the issues related to the multilateral teleoperation, we focus on two main questions: 1)How can we treat a multilateral system as a bilateral system?, and 2) Is there any mechanism that can be implemented to improve the transparency (i.e., to provide a faithful transmission of force and velocity) between the master and multiple-slaves in a 4-channels structure?

The facts that many works have been done in the past focusing on the bilateral teleoperations[4]-[6], then converting the multilateral teleoperation system to suit the existing bilateral structure, offers some advantages in terms of the controller design. Furthermore, as the total dynamics of slave manipulators can be changed (i.e., adding or subtracting slave manipulators, or to establish new configuration on the communication topology between agents), it is certainly cost inefficient if the dynamics of the master manipulator has also to be redesigned whenever the dynamics of slave manipulators have changed. Therefore, by introducing a single tunable equalizer to the master side renders flexibility to adjust the overall dynamics of the master manipulator to achieve symmetry between both sides of teleoperation. By this 
approach, the system designer requires only minimal knowledge on the dynamics of the newly-changed slave manipulators.

In this paper, we focus on the passive decomposition technique[7] to convert the multilateral teleoperation system into the bilateral form. Since the whole teleoperation process can be separated into two operations, namely grasping and handling, we use the sub-dynamics from the result of decomposition to determine the time (or in our case, ), that differentiates the time inverval between the two processes. In addition, we investigate the potential of introducing an equalizer connected to the master manipulator in order to achieve impedance matching between both sides of teleoperation.

Due to the orthonormal properties of the Laguerre function, the equalizer can be realized by a Laguerre network of high order of moderately damped system[8]. In most applications, the optimum structure of this network can be obtained by simply selecting the optimal values for the basis of the Laguerre filters[9]. The equalizer should be properly tuned to improve the overall performance. Hence, we adopted the so-called Fictitious-Reference-Iterative Tuning (FRIT) algorithm in our problem formulation. Unlike other tuning algorithms which requires mathematical model of the plant prior tuning, FRIT utilizes the measured input-and-output data to simultaneously attain the plant model and the optimum controller parameters[10]. Interestingly, it requires only a single-shot of measurement data for this purpose[11].

\section{PROBLEM FORMULATION}

\subsection{Notations and graph theory}

Let $\mathbb{R}$ and $\mathbb{R}^{m}$ denotes the set of real numbers and vectors of dimension $m$, respectively. Let $\mathbf{1}=[1, \ldots, 1]^{\top}$ be a vector with all elements of 1 and $\top^{\top}$ is the transposition. We use $\mathbf{I}_{n} \in \mathbb{R}^{n \times n}$ to denote the identity matrix. To denote the inner and cross products for $v, w \in \mathbb{R}^{p}$, we use $\langle v, w\rangle=v^{\top} w$ and $v \times w$, respectively. The vector norm is defined by $\|v\|:=\sqrt{v^{\top} v}$, and $\|v(k)\|_{K}^{2}$ implies

$$
\|v(k)\|_{K}^{2}:=\sum_{k=1}^{K}\|v(k)\|^{2}
$$

For $A=\left(a_{i j}\right) \in \mathbb{R}^{n \times m}$ and $B \in \mathbb{R}^{l \times k}$, the Kronecker product is defined by $A \otimes B=\left(a_{i j}\right) B \in \mathbb{R}^{n l \times m k}$. For $N$ number of agents, a graph $\mathbb{G}$ is built upon a finite set called a vertex set mapped by $\mathcal{V}=\left\{v_{1}, v_{2}, \ldots, v_{N}\right\}$ and the edge set of $\mathcal{E}$. We say $v_{i}, v_{j} \in \mathcal{E}$ if there is a communication link between them. Additionally, $\mathcal{N}_{i}=\left\{v_{j} \mid v_{i}, v_{j} \in \mathcal{E}, \forall j\right\}$ is the neighborhood set of $v_{i}$. The relationship between $v_{i}, v_{j} \in \mathcal{V}$ for an undirected graph $\mathbb{G}$ is encoded by the adjacency matrix $\mathcal{A}(\mathbb{G})=\left(a_{i j}\right) \in \mathbb{R}^{N \times N}$, where

$$
a_{i j}:= \begin{cases}1 & \text { if } v_{j} \in \mathcal{N}_{i} \\ 0 & \text { otherwise }\end{cases}
$$

The degree matrix $\mathcal{D}(\mathbb{G}) \in \mathbb{R}^{N \times N}$ is a diagonal matrix that $\mathcal{D}(\mathbb{G})=\operatorname{diag}(\mathcal{A}(\mathbb{G}) \mathbf{1}) \quad$ with $d_{i}:=\left|\mathcal{N}_{i}\right|=\sum_{j=1}^{N} a_{i j}$, and $\left|\mathcal{N}_{i}\right|$ is the cardinality of $\mathcal{N}_{i}$. The Laplacian matrix associated with $\mathbb{G}$ is defined by [12]:

$$
\mathcal{L}(\mathbb{G}):=\mathcal{D}(\mathbb{G})-\mathcal{A}(\mathbb{G}) \geq 0 .
$$

\subsection{Teleoperation architecture}

We consider a simple agent as the master manipulator governed by the equation of motion

$$
m_{m} \ddot{p}_{m}=f_{m}+f_{h} .
$$

Conversely, the slave manipulators depicted by a network of agents such that each agent $i$ moves dynamically through

$$
m_{i} \ddot{p}_{i}=f_{i}-f_{e_{i}} \forall i \in \mathbb{G} \text {. }
$$


The variables $m_{l} \in \mathbb{R}$, and $p_{l} \in \mathbb{R}^{2}$ are the agent's mass and position. The subscripts $l=m, i$ stand for the master and slave manipulators, respectively. The signals $f_{m} \in \mathbb{R}^{2}$ and $f_{h} \in \mathbb{R}^{2}$ are the exerted force of the master manipulator and the corresponding applied force of the human operator. Similarly, the signal $f_{i} \in \mathbb{R}^{2}$ is the exerted force of each agent $i$. The force $f_{e_{i}} \in \mathbb{R}^{2}$ is the estimated environment's force felt by each agent $i$ at its position relative to the center-of-mass (CoM) of the environment.

The object in the environment is considered to be a solid disk of radius $R$ that moves on a sliding flat surface. Assuming that all agents are friction-less (i.e., they move on friction-less wheels), the friction force only exists between the contact of the disk and the sliding surface. The desired final position of each agent $i$ on the environment is defined as

$$
\tilde{p}_{i}:=R\left[\cos \alpha_{i}(t) \sin \alpha_{i}(t)\right]^{\top}
$$

where $\alpha_{i}(t)=\alpha_{i}^{0}+\varphi(t)$ is the desired angular position of $i$, and $\alpha_{i}^{0}=2 \pi(i-1) / N \in \mathbb{R}, \forall i$. Meanwhile, $\varphi(t)$ is the environment's nominal angular displacement used in (7). For brevity, we drop the time notation $(t)$

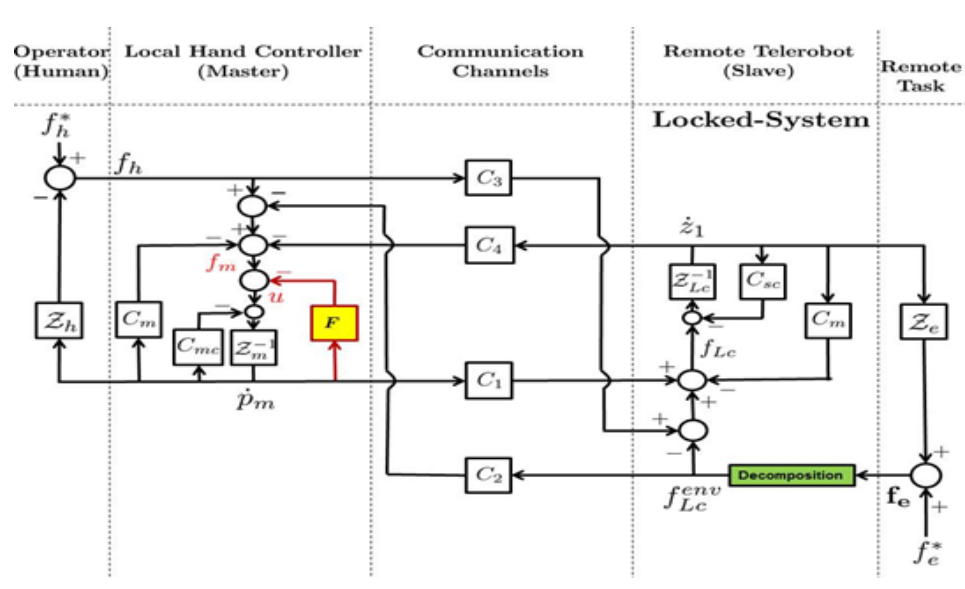

Figure 1 General Structure of Teleoperation Indicating the Equalizer $F$ and Intervenient Impedances, $C_{m s} \& C_{s c}$.

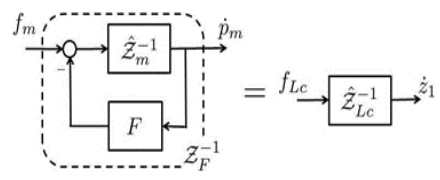

(a) Impedance Matching between Master and Locked System.

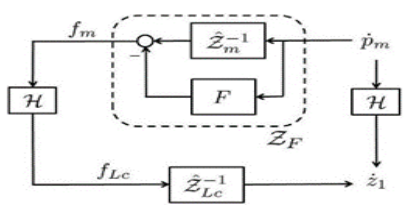

(b) Velocities Matching through $\mathcal{H}$.

Figure 2 Concept of Impedance Matching by FRIT.

in most equations, but use so whenever to emphasize its time-varying property. Defining $F_{f}$ as the friction force, the dynamical equation of the object is denoted by

$$
m_{e} \ddot{p}_{e}=\sum_{i=1}^{N} \tilde{f}_{i}-F_{f}, \quad \frac{1}{2} m_{e} R^{2} \ddot{\varphi}=\sum_{i=1}^{N} \tilde{\tau}_{i} .
$$

where $m_{e} \in \mathbb{R}$ and $p_{e} \in \mathbb{R}^{2}$ are the mass and CoM's position of the target/object, respectively. The force $\tilde{f}_{i} \in \mathbb{R}^{2}$ and torque $\tilde{\tau}_{i} \in \mathbb{R}$ in (7) are defined by

$$
\tilde{f}_{i}=\left\{\begin{array}{cl}
\frac{\left\langle f_{i},-\tilde{p}_{i}\right\rangle}{\left\langle-\tilde{p}_{i},-\tilde{p}_{i}\right\rangle}\left(-\tilde{p}_{i}\right) & \text { if }\left\|p_{i}-p_{e}\right\| \leq R \\
0 & \text { otherwise }
\end{array}\right.
$$

and

$$
\left[\begin{array}{c}
0 \\
0 \\
\tilde{\tau}_{i}
\end{array}\right]=\left\{\begin{array}{cl}
\left.\left[\begin{array}{c}
\tilde{p}_{i} \\
0
\end{array}\right] \times \begin{array}{c}
f_{i}-F_{f} \\
0
\end{array}\right] & \text { if }\left\|p_{i}-p_{e}\right\| \leq R \\
\mathbf{0} & \text { otherwise. }
\end{array}\right.
$$




\section{MULTILATERAL TO BILATERAL TELEOPERATION}

\subsection{Total Dynamics of the Slave Agents}

Following Kawakami \& Namerikawa[13] and to use (6), we introduce a change of variables as

$$
\hat{p}_{i}=p_{i}-\tilde{p}_{i}
$$

The force $f_{i}$ in (5) can be separated into two control components depending on the time interval, i.e., grasping and handling. We express

$$
f_{i}= \begin{cases}f_{i}^{f c}, & t<T_{\text {tele }}:(\text { grasp }) \\ f_{i}^{f c}+f_{i}^{t c}, & t \geq T_{\text {tele }}:(\text { grasp }+ \text { handle })\end{cases}
$$

where $f_{i}^{f c}$ and $f_{i}^{t c}$ are the formation and teleoperation control laws, respectively. The consensus control law

$$
f_{i}^{f c}=\gamma_{i}\left[a_{i e}\left\{-k\left(\hat{p}_{i}-p_{e}\right)-b\left(\dot{\hat{p}}_{i}-\dot{p}_{e}\right)\right\}+\sum_{j=1}^{N} a_{i j}\left\{-k\left(\hat{p}_{i}-\hat{p}_{j}\right)-b\left(\dot{\hat{p}}_{i}-\dot{\hat{p}}_{j}\right)\right\}\right]
$$

is used to achieve simultaneous grasping. Here $a_{i e}=1$ if $i$ can measure its relative positions and velocity with respect to object's CoM, and $a_{i e}=0$ otherwise. The gain $a_{i j}$ corresponds to the communication topology defined in (2), while the variables $k, b \in \mathbb{R}$ are the control gains. The gain $\gamma_{i} \in \mathbb{R}$ is defined by

$$
\gamma_{i}=\frac{1}{a_{i e}+\sum_{i=1}^{N} a_{i j}} .
$$

Substituting (8) and (10) into (5) and since $f_{e_{i}}=0$ for $t<T_{t e l e}$, we now rewrite the dynamics of agent $i$ as

$$
m_{i} \ddot{\hat{p}}_{i}=\gamma_{i}\left[a_{i e}\left\{-k\left(\hat{p}_{i}-p_{e}\right)-b\left(\dot{\hat{p}}_{i}-\dot{p}_{e}\right)\right\}+\sum_{j=1}^{N} a_{i j}\left\{-k\left(\hat{p}_{i}-\hat{p}_{j}\right)-b\left(\dot{\hat{p}}_{i}-\dot{\hat{p}}_{j}\right)\right\}\right] .
$$

Suppose the target object acts as the virtual $(N+1)$ th agents, then we have $p_{e}=\hat{p}_{N+1}=p_{N+1}-\tilde{p}_{N+1}$ with $\tilde{p}_{N+1}=0$ and $a_{i e}=a_{i(N+1)}$. In addition, the mass of the virtual agent is to take the average mass of all agents given by $m_{N+1}=\frac{1}{N} \sum_{i=1}^{N} m_{i}$. By this, (12) can be written as

$$
\frac{m_{i}}{\gamma_{i}} \ddot{\hat{p}}_{i}=-k \sum_{j=1}^{N+1} a_{i j}\left(\hat{p}_{i}-\hat{p}_{j}\right)-b \sum_{j=1}^{N+1} a_{i j}\left(\dot{\hat{p}}_{i}-\dot{\hat{p}}_{j}\right)
$$

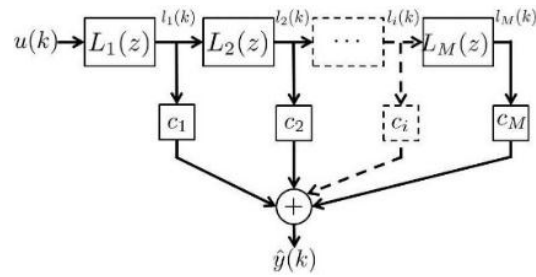

Figure 3. Structure of the Laguerre Network

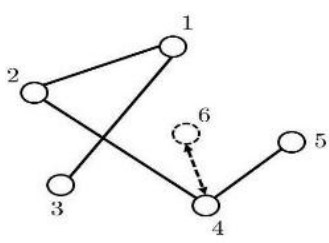

Figure 4. Communication Topology between Agents (No.6 is the Virtual Agent Representing the Target Object)

The total dynamic consisting of $N+1$ agents is given by

$$
\mathbf{M} \ddot{\hat{\mathbf{p}}}+b\left(\hat{\mathcal{L}} \otimes \mathbf{I}_{2}\right) \dot{\hat{\mathbf{p}}}+k\left(\hat{\mathcal{L}} \otimes \mathbf{I}_{2}\right) \hat{\mathbf{p}}= \begin{cases}\mathbf{0}, & t<T_{\text {tele }} \\ \mathbf{f}-\mathbf{f}_{e}, & t \geq T_{\text {tele }}\end{cases}
$$


where $\left.\hat{\mathbf{p}}=\left[\hat{p}_{1}^{\top}, \ldots, \hat{p}_{N+1}^{\top}\right]^{\top} \in \mathbf{R}^{2(N+1)}, \mathbf{f}=\left[f_{1}^{t c}{ }^{\top}, \ldots, f_{N+1}^{t c}\right]^{\top}\right]^{\top}, \mathbf{f}_{e}=\left[f_{e_{1}}^{\top}, \ldots, f_{e_{N+1}}^{\top}\right]^{\top}$, and

$\mathbf{M}:=\operatorname{diag}\left(m_{1} / \gamma_{1}, \cdots, m_{(N+1)} / \gamma_{N+1}\right) \otimes \mathbf{I}_{2}$.

Meanwhile, $\hat{\mathcal{L}}$ is the Laplacian matrix composed of the assorted actual and virtual slave agents defined by $\hat{\mathcal{L}}=\left(\hat{l}_{i j}\right) \in \mathbb{R}^{(N+1) \times(N+1)}$ where

$$
\hat{l}_{i j}:= \begin{cases}\sum_{k=1}^{N+1} a_{i k} & \text { if } i=j \\ -a_{i j} & \text { if } i \neq j .\end{cases}
$$

\subsection{Passive Decomposition[7] in Handling}

Introduce a new coordinate system in the form of

$$
\mathbf{z}=\left[z_{1}^{\top}, z_{2}^{\top}, \ldots, z_{N+1}^{\top}\right]^{\top}:=S \hat{\mathbf{p}}
$$

where $S$ is the transformation matrix defined by

$$
\begin{aligned}
S:=\left[\begin{array}{ccccc}
\hat{\mathbf{M}}_{1} & \hat{\mathbf{M}}_{2} & \ldots & \hat{\mathbf{M}}_{N} & \hat{\mathbf{M}}_{N+1} \\
\mathbf{I} & \mathbf{0} & \ldots & \mathbf{0} & -\mathbf{I} \\
\mathbf{0} & \mathbf{I} & \ddots & \mathbf{0} & -\mathbf{I} \\
\vdots & \ddots & \ddots & \vdots & \vdots \\
\mathbf{0} & \mathbf{0} & \ldots & \mathbf{I} & -\mathbf{I}
\end{array}\right] \in \mathbb{R}^{n(N+1) \times n(N+1)}, \\
\hat{\mathbf{M}}_{j}=\frac{m_{j}}{m_{L} \gamma_{j}} \mathbf{I}_{2}, j=1, \ldots, N+1, \quad m_{L}=\sum_{i=1}^{N+1} \frac{m_{i}}{\gamma_{i}}
\end{aligned}
$$

The sub-coordinate $z_{1} \in \mathbb{R}^{2}$ corresponds to the Locked system's coordinate. Accordingly, $\mathbf{z}_{e}=\left[z_{2}^{\top}, \ldots, z_{N+1}^{\top}\right]^{\top} \in \mathbb{R}^{2 N}$ represents the Shape system's coordinate[7]. Substituting (17) into (14), we obtain the total dynamics in the new coordinate system as

$$
S^{-\top} \mathbf{M} S^{-1} \ddot{\mathbf{z}}+b S^{-\top}\left(\hat{\mathcal{L}} \otimes \mathbf{I}_{2}\right) S^{-1} \dot{\mathbf{z}}+k S^{-\top}\left(\hat{\mathcal{L}} \otimes \mathbf{I}_{2}\right) S^{-1} \mathbf{z}=S^{-\top}\left(\mathbf{f}-\mathbf{f}_{e}\right) .
$$

With this transformation, it is observed that the mass matrix (19) is block-diagonalized such that

$$
S^{-\top} \mathbf{M} S^{-1}=:\left[\begin{array}{cc}
m_{L} \mathbf{I}_{2} & \mathbf{0} \\
\mathbf{0} & \overline{\mathbf{M}}
\end{array}\right]
$$

where $\overline{\mathbf{M}}=\overline{\mathbf{M}}^{\top} \succ 0 \in \mathbf{R}^{2 N \times 2 N}$. Similarly, the information graph (16) is decomposed to attain

$$
S^{-\top}\left(\hat{\mathcal{L}} \otimes \mathbf{I}_{2}\right) S^{-1}=\left[\begin{array}{cc}
\mathbf{0}_{2 \times 2} & \mathbf{D}^{\top} \\
\mathbf{0}_{N \times 2} & \overline{\mathbf{L}}
\end{array}\right],
$$

where the structures of $\mathbf{D} \in \mathbb{R}^{2 N \times 2}$ and $\overline{\mathbf{L}} \in \mathbb{R}^{2 N \times 2 N}$ are identical to the one defined by Lee \& Spong[7]. The Locked and Shape systems of the total dynamics (14) as $t \geq T_{t e l e}$ are obtained as

$$
\begin{aligned}
m_{L} \ddot{z}_{1}+b \mathbf{D}^{\top} \dot{\mathbf{z}}_{e}+k \mathbf{D}^{\top} \mathbf{z}_{e} & =f_{L c}-f_{L c}^{e n v} \\
\overline{\mathbf{M}} \ddot{\mathbf{z}}_{e}+b \overline{\mathbf{L}} \dot{\mathbf{z}}_{e}+k \overline{\mathbf{L}} \mathbf{z}_{e} & =f_{S h}-f_{S h}^{e n v}
\end{aligned}
$$

where $\left[f_{L c}^{\top}, f_{S h}^{\top}\right]^{\top}=S^{-\top} \mathbf{f}$, and $\left[f_{L c}^{e n v}{ }^{\top}, f_{S h}^{e n v}\right]^{\top}=S^{-\top} \mathbf{f}_{e}$. Notice that the Locked system (22) is coupled with the Shape system (23) through D. In one approach, Nam \& Namerikawa[14] introduced a decoupling control input into the system to eliminate the coupled term. However, Lee \& Spong[7] emphasized that D 
can be eliminated through proper selection of graph topology (3). Following result in [7], the Locked system (22) became the newly decoupled Locked system defined by

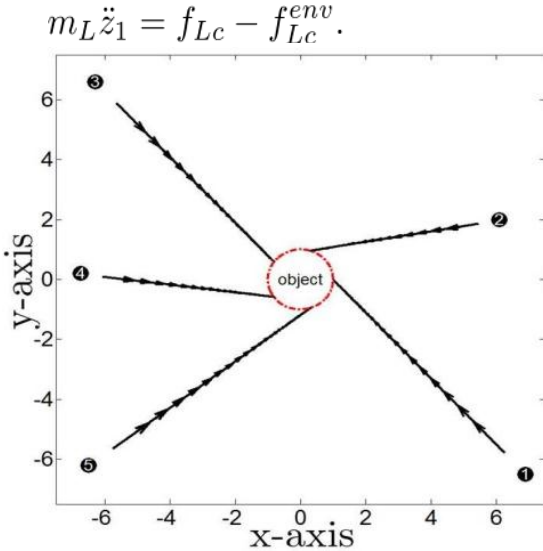

(a)
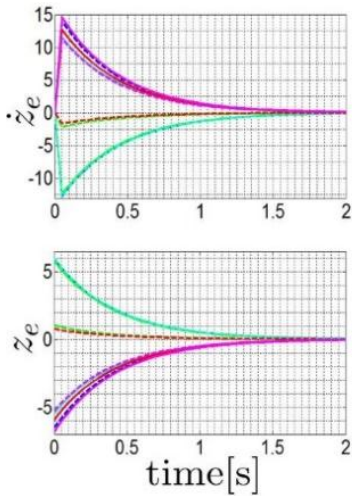
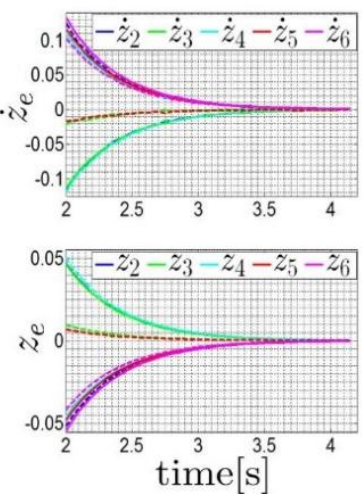

(b)

Figure 5. Asymptotic Convergence of the Agents onto Their Intended Final Positions on the Target Object as $t<T_{t e l e}$, with $\varphi(0)=0$. (a) Agent's Trajectories, (b) Velocity and Position of the Shape System.

The following lemma provides the sufficient conditions for selecting gains $k, b$ to ensure that all slave agents simultaneously arrive at the intended final targets by the consensus control law (10).

Lemma 1. Suppose for the total dynamics of the slave agents in (14) and $t<T_{\text {tele }}$, there exist gains $k, b>0$ to guarantee simultaneous asymptotic convergence of all slave agents to their intended final positions on the environment/target iff the following conditions are met

$$
2 b^{2} \overline{\mathbf{L}}_{s y m}-k \overline{\mathbf{M}} \succ 0 \text { and } \overline{\mathbf{L}}_{s y m} \succ 0,
$$

where $\overline{\mathbf{L}}_{\text {sym }}=\frac{1}{2}\left(\overline{\mathbf{L}}+\overline{\mathbf{L}}^{\top}\right)$ is the symmetric component of $\overline{\mathbf{L}}$.

\subsection{Tele-operation time $T_{\text {telle }}$}

The estimation of $T_{\text {tele }}$ is crucial since it is considered as the waiting time by the master manipulator before the handling session begins. This parameter is non-unique since it depends on the positions and velocities of all slave agents at $t=0$.

Theorem 1. Suppose for any ${ }^{k}$ and ${ }^{b}$ satisfying Lem.1, with any arbitrary initial positions $\mathbf{p}(0) \in \mathbb{R}^{2 N}$ and velocities $\dot{\mathbf{p}}(0) \in \mathbb{R}^{2 N}$ of the agents, then the approximate "maximum" time $T_{\text {tele taken by }}$ the agents to reach to the intended final positions on the environment such that for a small scalar $\tilde{\epsilon}>0$, $\|x\|<\tilde{\epsilon}$ for $t>T_{\text {tele. }}$.

Proof. As for $t<T_{\text {tele, we have }} f_{S h}-f_{S h}^{e n v}=0$. Then the Shape system (23) can be written in the form of autonomous LTI system of $\dot{x}(t)=A x(t)$ where $x=\left[\dot{\mathbf{z}}_{e}^{\top}, \mathbf{z}_{e}^{\top}\right]^{\top} \in \mathbb{R}^{2 N}$, and $A$ is a stable matrix. Hence, there exist $\bar{a}>0$ and $\bar{k}>0$ such that $\left\|e^{A t}\right\| \leq \bar{k} e^{-\bar{a} t}$. Then,

$$
\|x(t)\|=\left\|e^{A t} x(0)\right\| \leq\left\|e^{A t}\right\|\|x(0)\| \leq \bar{k} e^{-\bar{a} t}\|x(0)\| .
$$

Hence, when $t>\frac{1}{\bar{a}} \ln \bar{k}\|x(0)\| / \tilde{\epsilon},\|x(t)\| \leq \tilde{\epsilon}$. Therefore, $T_{\text {tele }}=\frac{1}{\bar{a}} \ln \bar{k}\|x(0)\| / \tilde{\epsilon}$.

\section{IMPEDANCE MATCHING BY FRIT}

Consider the time interval $t>T_{\text {tele }}$, and based on the block diagram in Figure 1, the following control laws for the sub-dynamics of (24) and (23) are used: 


$$
\begin{aligned}
f_{L c} & =C_{1} \dot{p}_{m}-C_{m} \dot{z}_{1}+C_{3} f_{h}, \\
f_{S h} & =f_{S h}^{e n v} .
\end{aligned}
$$

Rewriting the dynamics of the master and slave (Locked) manipulators in terms of their impedance yields us to

$$
\dot{p}_{m}=\mathcal{Z}_{m}^{-1} f_{m} \quad \text { and } \quad \dot{z}_{1}=\mathcal{Z}_{L c}^{-1} f_{L c}
$$

where $\mathcal{Z}_{m}=\mathbf{M}_{m} s$ and $\mathcal{Z}_{L c}=\mathbf{M}_{L} s$, respectively. An equalizer is placed in the feedback loop of the master manipulator. By optimally tuning this equalizer will symmetrize the impedance in both sides of teleoperation. Contrary to Tsuji et.al.[15], we consider a special structure of $F$ and the tuning procedure is carried-out using the 2 -dimensional space data. Figure 1 also illustrates the placement of the equalizer $F$ in the general structure of our teleoperation system. The control gains $C_{1}, C_{2}, C_{3}$ and $C_{4}$ can be designed accordingly to form either "position-position", "position-force" or "optimized" teleoperation architectures[16]. To avoid acceleration measurement, additional controllers $C_{m c}$ and $C_{s c}$ (low-gain PD Control) are employed as the intervenient impedance to the original systems[16]. Hence, (29) is rewritten as

$$
\dot{p}_{m}=\hat{\mathcal{Z}}_{m}^{-1} f_{m} \quad \text { and } \quad \dot{z}_{1}=\hat{\mathcal{Z}}_{L c}^{-1} f_{L c}
$$

where $\hat{\mathcal{Z}}_{m}=\mathcal{Z}_{m}+C_{m c}$ and $\hat{\mathcal{Z}}_{L c}=\mathcal{Z}_{L c}+C_{s c}$.

\subsection{Fictitious Reference Iterative Tuning (FRIT)}

FRIT is a simple yet powerful tuning algorithm in the sense that we require only a single-shot of experimental data of the closed-loop system to obtain the optimum controller parameters[10]. Figure 2 depicts the main concepts of impedance matching using FRIT in Tsuji et.al. [15]. As illustrated in Figure 3(a), the ultimate aim of introducing the equalizer $F$ is to achieve $\hat{\mathcal{Z}}_{F} \rightarrow \hat{\mathcal{Z}}_{L c}$. However, before $F$ can be tuned, it is necessary to match the velocities of the master and the Locked system through $\mathcal{H}_{\text {as depicted in }}$ Figure 3(b). Hence, we solve the optimization problem:

$$
\min _{\mathcal{H}} J_{H}
$$

where for the recorded initial data $\dot{p}_{m}^{0}(k)$ and $\dot{z}_{1}^{0}(k)$,

$$
J_{H}:=\left\|\dot{z}_{1}^{0}(k)-\mathcal{H}(z) \dot{p}_{m}^{0}(k)\right\|_{K}^{2} .
$$

Based on the optimal $\mathcal{H}(z)$, we solve the second optimization problem:

$$
\min _{F} J_{F}
$$

where for the recorded initial data $f_{m}^{0}(k)$ and $f_{L c}^{0}(k)$ when the initial equalizer $F_{0}(z)$ is used,

$$
J_{F}:=\left\|f_{L c}^{0}(k)-\tilde{f}_{L c}(k)\right\|_{K}^{2},
$$

and the fictitious reference signal is

$$
\begin{aligned}
\tilde{f}_{L c}(k) & :=\mathcal{H}(z)\left(u^{0}(k)+F(z) \dot{p}_{m}^{0}(k)\right), \\
u^{0}(k) & =f_{m}^{0}(k)-F_{0}(z) \dot{p}_{m}^{0}(k) .
\end{aligned}
$$

Theorem 2. Suppose that $\mathcal{H}^{*}:=\arg \min _{\mathcal{H}} J_{H}$ and $F^{*}:=\arg \min _{F} J_{F}$ attain $J_{H}=0$ and $J_{F}=0$,respectively. Then, if the signal $\dot{\varkappa}_{1}^{0}(k)$ is rich,

$$
\hat{\mathcal{Z}}_{F}=\hat{\mathcal{Z}}_{m}+F^{*}=\hat{\mathcal{Z}}_{L c} .
$$




\subsection{Laguerre network}

We can approximate a discrete time SISO system to use a Laguerre function

$$
L_{i}(z)=\beta \frac{(1-\alpha z)^{i-1}}{(z-\alpha)^{i}},
$$

as $\hat{y}(z)=g(z) u(z)=\sum_{i=1}^{M} c_{i} L_{i} u(z)$ (Figure 3). Here $\alpha:=e^{-p T}$ and $\beta:=\sqrt{\left(1-\alpha^{2}\right) T}$. The parameter $p$ is determined by the system's designer and $T$ is the sample time. Meanwhile $c_{i} \in \mathbb{R}, i=1, \ldots, M$ are the coefficients that form the basis of the Laguerre network. Let $l_{i}(k) \in \mathbb{R}, i=1, \ldots, M$ represents the output of the $i$ th-order filter in the Laguerre network. Then, the SISO state-space model is given by

$$
g(z):\left\{\begin{array}{l}
l(k+1)=a l(k)+b u(k) \\
\hat{y}(k)=c^{T} l(k)
\end{array}\right.
$$

where $l=\left[l_{1}, \ldots, l_{M}\right]^{T} \in \mathbb{R}^{M}, a \in \mathbb{R}^{M \times M}, b \in \mathbb{R}^{M}, c \in \mathbb{R}^{M}$,

$$
\begin{aligned}
& \{a\}_{i j}:= \begin{cases}\frac{(-1)^{i-j} \tau_{2}^{i-j-1}\left(\tau_{1} \tau_{2}+\tau_{3}\right)}{T^{i-j}} & \begin{array}{l}
\text { if } i=j \\
0
\end{array} \\
\text { if } i>j & \text { otherwise, }\end{cases} \\
& \{b\}_{i}:=\left(-\frac{\tau_{2}}{T}\right)^{i-1} \tau_{4} \\
& c^{T}=\left[\begin{array}{lll}
c_{1} & \ldots & c_{M}
\end{array}\right] . \\
& \tau_{1}=e^{-p T}, \quad \tau_{2}=T+\frac{2}{p}\left(e^{-p T}-1\right), \tau_{3}=-T e^{-p T}-\frac{2}{p}\left(e^{-p T}-1\right), \tau_{4}=\frac{\sqrt{2 p}}{p}\left(1-\tau_{1}\right) .
\end{aligned}
$$

The MIMO transfer matrix $G(z)=\mathbf{I}_{n} \otimes g(z)$ has a state-space representation given as

$$
G(z):\left\{\begin{array}{l}
\mathbf{l}(k+1)=\mathbf{A l}(k)+\mathbf{B} u(k) \\
\hat{y}(k)=\mathbf{C}^{T} \mathbf{l}(k)
\end{array}\right.
$$

where $\mathbf{l}=\mathbf{1}_{n} \otimes l \in \mathbb{R}^{n M}, \mathbf{A}=\mathbf{I}_{n} \otimes a \in \mathbb{R}^{n M \times n M}, \mathbf{B}=\mathbf{I}_{n} \otimes b \in \mathbb{R}^{n M \times n}$ and $\mathbf{C}^{T}=\mathbf{I}_{n} \otimes c^{T} \in \mathbb{R}^{n \times n M}$. The appropriate design of the equalizer $F$ can be obtained by assigning (44) into $F$. To ensure faster rate convergence of the Laguerre network, Wahlberg[17] suggested that the value of $p$ in (43) should be chosen close to the inverse of the system dominant time constant. However in our case, the parameter of $p$ must be chosen so that the designed equalizer will be in-sync with the Shape system (23). Hence, the parameter $p$ is to use the parameter's value of $\bar{a}_{\text {in }}(26)$ such that $p=\bar{a}$. Therefore, we are left with the tunable parameters of $c$, where the optimum equalizer $F$ can be obtained by simply tuning the basis (42).

The selection of the equalizer $\mathcal{H}$ to solve the optimization problem of (31), requires $\mathcal{H}$ to be inverse-able and $\mathcal{H}^{-1}$ is stable. Therefore, we select a biproper transfer function in the form of

$$
h(z)=\frac{1+\hat{a}_{1} z^{-1}+\cdots+\hat{a}_{P} z^{-P}}{1+\hat{b}_{1} z^{-1}+\cdots+\hat{b}_{P} z^{-P}}
$$

to yield us a MIMO transfer matrix of $\mathcal{H}=\mathbf{I}_{n} \otimes h(z)$. The estimation of the coefficients $\hat{a}_{i}, \hat{b}_{i} \in \mathbb{R}$ in (45) can be obtained by minimizing (32) through least-square method (LSM). Re-arranging data such that 


$$
\begin{aligned}
\Psi_{H}= & {\left[\begin{array}{ccc:ccc}
\dot{p}_{m}^{0}(P) & \cdots & \dot{p}_{m}^{0}(1) & -\dot{z}_{1}^{0}(P) & \cdots & -\dot{z}_{1}^{0}(1) \\
\vdots & \cdots & \vdots & \vdots & \cdots & \vdots \\
\dot{p}_{m}^{0}(K-1) & \cdots & \dot{p}_{m}^{0}(K-P) & -\dot{z}_{1}^{0}(K-1) & \cdots & -\dot{z}_{1}^{0}(K-P)
\end{array}\right] \in \mathbb{R}^{n(K-P) \times n P}, } \\
\Upsilon_{H}= & {\left[\begin{array}{c}
\dot{z}_{1}^{0}(P+1)-\dot{p}_{m}^{0}(P+1) \\
\vdots \\
\dot{z}_{1}^{0}(K)-\dot{p}_{m}^{0}(K)
\end{array}\right] \in \mathbb{R}^{n(K-P)}, }
\end{aligned}
$$

and $\chi_{H}=\left[\begin{array}{llllll}\hat{a}_{1} & \cdots & \hat{a}_{P} & \hat{b}_{1} & \cdots & \hat{b}_{P}\end{array}\right]^{\top} \in \mathbb{R}^{n P}$, to give us a linear relation of $\Psi_{H} \chi_{H}=\Upsilon_{H}$. Then, the optimal coefficients are obtained by

$$
\chi_{H}^{*}=\arg \min _{\chi_{H}} J_{H}=\Psi_{H}^{\dagger} \Upsilon_{H}
$$

where $\Psi_{H}^{\dagger}:=\left(\Psi_{H}^{\top} \Psi_{H}\right)^{-1} \Psi_{H}^{\top}$ is the Moore-Penrose pseudoinverse of $\Psi_{H}$. Next, we illustrate the tuning procedure to obtain the optimum equalizer $F$. The cost function (34) is simplified to

$$
J_{F}=\left\|f_{L c}^{0}-\mathcal{H}^{*}\left(u^{0}(k)+F(z)\right) \dot{p}_{m}^{0}(k)\right\|_{K}^{2}=\left\|\left(\mathcal{H}^{*-1} f_{L c}^{0}(k)-u^{0}(k)\right)-F(z) \dot{p}_{m}^{0}(k)\right\|_{K}^{2}
$$

where $\mathcal{H}^{*}$ is $\mathcal{H}$ with the optimized parameters obtained by (46). The overall algorithm to obtain optimum $F$ is summarized as following:

Step 1: Set a Laguerre network $F^{0}(z)$ using (63) and $\mathbf{C}_{0}=\mathbf{I}_{n} \otimes c_{0} \in \mathbb{R}^{n \times n M}$ with an appropriate initial $c=c_{0}$ as

$$
F^{0}(z):\left\{\begin{array}{l}
\mathbf{l}(k+1)=\mathbf{A l}(k)+\mathbf{B} \dot{p}_{m}(k) \\
\hat{y}_{L}(k)=\mathbf{C}_{0}^{\top} \mathbf{l}(k) .
\end{array}\right.
$$

Step 2: Perform an experiment to generate the initial data $\dot{p}_{m}^{0}, \dot{z}_{1}^{0}, f_{L c}^{0}$ and $f_{m}^{0}$.

Step 3: Choose a bi-proper transfer function $\mathcal{H}$, and minimize (32) by (46) using data obtained in Step 2.

Step 4: Using the optimally tuned $\mathcal{H}(z)$, find the minimizer $F^{*}(z)$ to minimize the cost function (47). The sub-algorithm in Step 4 to tune $F$ is as follows:

Step 1 : Generate the new data $\tilde{y}(k)=\mathcal{H}^{*-1} f_{L c}^{0}(k)-u^{0}(k)$.

Step 2 : Using $\dot{p}_{m}^{0}$ in Step 2, generate the state data $\mathbf{l}(k)$.

Step 3: $\quad$ Find the minimizer $c^{*}$ of the constraint minimization

$$
\begin{aligned}
& \min _{c}\left\|\mathbf{C}^{T} \mathbf{l}(k)-\tilde{y}(k)\right\|_{K}^{2}+\eta\left\|c-c_{0}\right\|^{2} \\
& \text { subject to } \mathbf{C}^{T}=\mathbf{I}_{n} \otimes c^{T} \in \mathbb{R}^{n \times n M} .
\end{aligned}
$$

This is equivalent to

$$
\min _{c} \sum_{i=1}^{n}\left\|c^{T} l(k)-\tilde{y}_{i}(k)\right\|_{K}^{2}+\eta\left\|c-c_{0}\right\|^{2} .
$$

Then, the minimizer is obtained by

$$
c^{T *}=\Upsilon_{F} \Psi_{F}^{T}\left(\Psi_{F} \Psi_{F}^{T}\right)^{-1}
$$

where

$$
\begin{aligned}
& \Psi_{F}=\left[l(P+1), \cdots, l(K), l(P+1), \cdots, l(K) \cdots, l(P+1), \cdots, l(K), \sqrt{\eta} I_{M}\right] \in \mathbb{R}^{M \times(n(K-P)+M)} \\
& \Upsilon_{F}=\left[\tilde{y}_{1}(P+1), \cdots, \tilde{y}_{1}(K), \tilde{y}_{2}(P+1), \cdots, \tilde{y}_{2}(K), \tilde{y}_{n}(P+1), \cdots, \tilde{y}_{n}(K), c_{0}^{T}\right] \in \mathbb{R}^{1 \times(n(K-P)+M)} .
\end{aligned}
$$




\section{NUMERICAL EXAMPLE}

A numerical example to illustrate the effectiveness of our approach is presented in this section. Let the slave manipulators comprised of $N=5$ agents with their communication topology as shown in Figure 4. The dashed-arrow indicates that only agent 4 can measure the position and velocity of the target object (indicated by virtual agent 6 ). The mass of the master, slave agents and the environment were chosen as $\left[m_{m}, m_{i}, m_{e}\right]=[2.0,0.5,10] \mathrm{kg}, \forall i$. Meanwhile, the radius of the target object/environment was chosen as
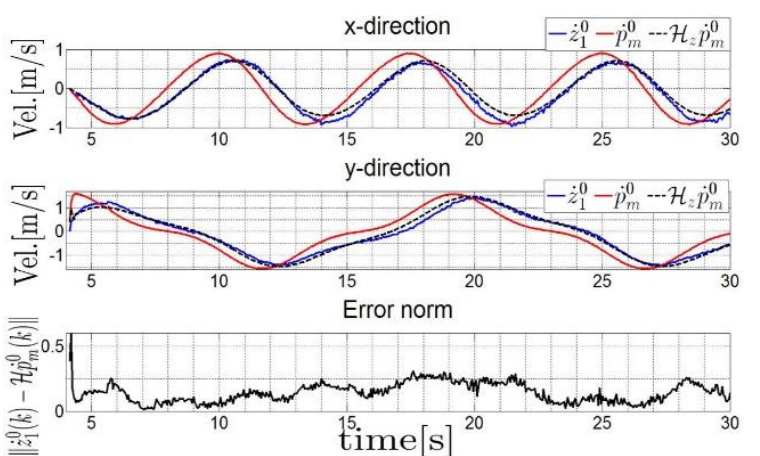

Figure 6 Velocity Matching Through Gain
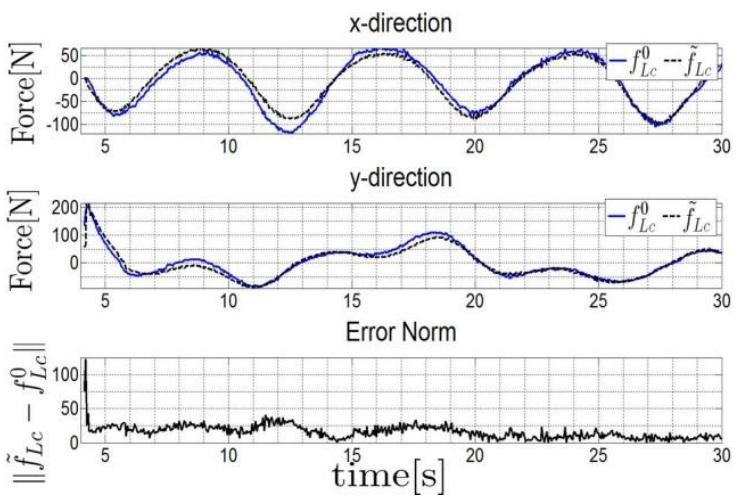

Figure 7 Comparison of Optimized Fictitious Signal (35) with the Initial $f_{L c}$

$R=1 \mathrm{~m}$. To satisfy Lemma 1 , the spring and damping constants in (12) were selected as $[k, b]=[1200 \mathrm{~N} / \mathrm{m}, 500 \mathrm{Ns} / \mathrm{m}]$. With this selection, the dominant eigenvalue of the Shape system (23) has been obtained as $\lambda_{\text {tele }}=\lambda_{\min }=2.4029$. For ease of analysis, we assume that the friction force, $F_{f}=0$.

The initial positions of the master manipulator and the C.O.M of the target(environment) were set at $(0,0)$ and being stationary at $t \in\left[0, T_{\text {tele }}\right)$. Meanwhile, the slave agents' positions $p_{i}(0) \in \mathbb{R}^{2}, \forall i$ were initialized around the target in the region $[-10,10] \times[-10,10] \subseteq \mathbb{R}^{2}$, with zero velocity at $t=0$ i.e., $\dot{p}_{i}(0)=0, \forall i$. In Figure $5(\mathrm{a})$, the agents' initial positions and trajectories towards their intended final positions on the object for $t \in\left[0, T_{\text {tele }}\right)$ are illustrated. Subsequently, Figure 5(b) depicts the trajectory of Shape system's velocity and position. It is clearly observed that the trajectories of the Shape system were asymptotically converging such that $\lim _{t \rightarrow T_{\text {tele }}} \mathbf{z}_{e}=\lim _{t \rightarrow T_{\text {tele }}} \dot{\mathbf{z}}_{e}=0$. The estimated teleoperation time based on the given initial positions were obtained as $T_{\text {tele }}=4.1331 \mathrm{~s}$. In this example, we used the intervenient impedances $C_{m c}=\mathbf{I}_{2} \otimes\left(2+\frac{20}{s}\right), C_{s c}=\mathbf{I}_{2} \otimes\left(20+\frac{6}{s}\right)$, the controller $C_{m}=\mathbf{I}_{2} \otimes\left(100+\frac{1}{s}\right)$, with human impedance of $\mathcal{Z}_{h}=\mathbf{I}_{2} \otimes\left(0.5 s+70+\frac{2000}{s}\right), \eta=0.1$, and $T_{\text {sim }}=30[\mathrm{sec}]$, respectively.

The velocity matching performance between $\mathcal{H} \dot{p}_{m}(k)$ and $\dot{z}_{1}(k)$ as presented in Figure 6 verifies

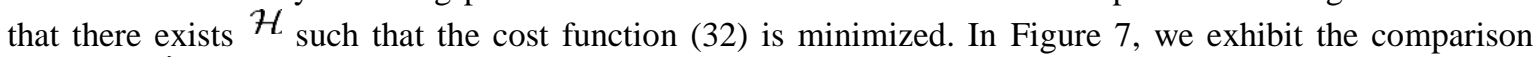
between $f_{L c}^{0}(k)$ and the response of fictitious signal (35) when the equalizer $F$ with optimally tuned parameters was applied. It can be shown that these two signals were almost identical in both $x$ and $y$ directions in the sense that $\left\|\tilde{f}_{L c}(k)-f_{L c}^{0}(k)\right\|_{\text {is minimized. }}$

\section{CONCLUSION}

In this paper, we have proposed tuning algorithm to improve transparency through impedance matching between master and slave manipulators in the multilateral teleoperation system. The passive decomposition technique has provided substantial approach to convert the total dynamics of a multi-slave system into the bilateral teleoperation architecture so that the existing bilateral control technique can be used. Subsequently, from the result of decomposition and the initial states of all slave agents, the teleoperation time, $T_{\text {tele }}$ that separated the two operations (grasping and teleoperation) has been estimated. In terms of obtainining the optimal equalizer, we conclude that FRIT provides powerful algorithms since only a singleshot of measurement data was required for tuning. The overall performance and comparison presented through numerical example has illustrated the applicability of the proposed tuning method. 


\section{ACKNOWLEDGEMENTS}

The authors would like to thank Universiti Malaysia Pahang for their full support in this research by providing the financial assistance through the short-term grant RDU1703139. Special thank also goes to Professor Dr. Shigeru Yamamoto from MoCCoS Laboratory, Kanazawa University, Japan, for the insightful discussion on the mathematical derivation.

\section{REFERENCES}

[1] L. Marton, Z. Szanto, P. Haller, H. Sandor, and T. Szabo, "Bilateral teleoperation of wheeled mobile robots working in common workspace," IAES International Journal of Robotics and Automation (IJRA), vol. 3, no. 1, pp. 62-74, 2014.

[2] D. H. Zhai and Y. Xia, "Adaptive fuzzy control of multilateral asymmetric teleoperation for coordinated multiple mobile manipulators," IEEE Trans. Fuzzy Syst., vol. 24, no. 1, pp. 57-70, 2016.

[3] Y. Kawai, T. Miyoshi, and M. Fujita, "Written Communication System based on Multilateral Teleoperation using Robust Control,” in IEEE International Conference on Advanced Intelligent Mechatronics, 2017, pp. 657-662.

[4] P. F. Hokayem and M. W. Spong, "Bilateral teleoperation: An historical survey," Automatica, vol. 42, no. 12, pp. 2035-2057, Dec. 2006.

[5] G. Wei and Z. Miaolei, "Fuzzy PID control method for internet-based tele-operation manipulators system," IAES Telkomnika, vol. 11, no. 11, pp. 6381-6389, 2013.

[6] L. Marton, Z. Szanto, P. Haller, H. Sandor, T. Szabo, and T. Vajda, "Bilateral Teleoperation of Wheeled Mobile Robots Working in Common Workspace," IAES Int. J. Robot. Autom., vol. 3, no. 1, pp. 61-74, 2014.

[7] D. Lee and M. W. Spong, "Stable flocking of multiple inertial agents on balanced graphs," IEEE Trans. Autom. Control, vol. 52, no. 8, pp. 1469-1475, 2007.

[8] Q. G. Zhou and E. J. Davison, "A simplified algorithm for balanced realization of Laguerre network models," in Proceedings of the 39th IEEE Conference on Decision and Control, 2000, vol. 5.

[9] T. O. e Silva, "Optimality conditions for truncated Laguerre networks," IEEE Trans. Signal Process., vol. 42, no. 9, pp. 2528-2530, 1994.

[10] O. Kaneko, Y. Yamashina, and S. Yamamoto, "Fictitious reference tuning for the optimal parameter of a feedforward controller in the two-degree-of-freedom control system," Proc. IEEE Int. Conf. Control Appl., vol. 4, no. 1, pp. 59-64, 2010.

[11] H. T. Nguyen, O. Kaneko, and S. Yamamoto, "Data-driven IMC for Non-Minimum Phase Systems - Laguerre Expansion Approach -," in 50th IEEE Conference on Decision and Control and European Control Conference, 2011, pp. 476-481.

[12] M. Mesbahi and M. Egerstedt, Graph theoretic methods in multiagent networks. Princeton University Press, 2010.

[13] H. Kawakami and T. Namerikawa, "Cooperative target-capturing strategy for multi-vehicle systems with dynamic network topology," in American Control Conference, 2009, pp. 635-640.

[14] D. Do Nam, Y. Yamashina, and T. Namerikawa, "Bilateral teleoperation of multiple cooperative robots with time-varying delay, ” 2010 IEEE Int. Conf. Control Appl., pp. 2053-2058, Sep. 2010.

[15] M. Tsuji, S. Yamamoto, and O. Kaneko, "A tuning method of a 4-channel bilateral control system," in 46th SICE Hokkaido Branch Academic Symposium (in Japanese), 2014, no. 4, pp. 1-4.

[16] M. Zhu and S. E. Salcudean, "Achieving transparency for teleoperator systems under position and rate control," Proc. 1995 IEEE/RSJ Int. Conf. Intell. Robot. Syst. Hum. Robot Interact. Coop. Robot., vol. 2, pp. 7-12, 1995.

[17] B. Wahlberg, "System identification using Laguerre models," IEEE Trans. Automat. Contr., vol. 36, no. 9143255, pp. 551-562, 1991. 


\section{BIOGRAPHIES OF AUTHORS}

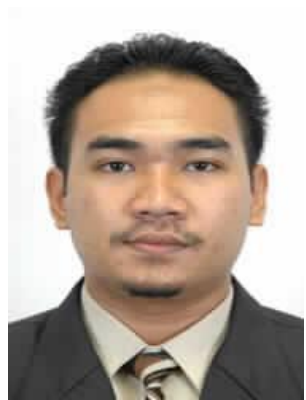

Mohd Syakirin Ramli received the B. Sc. degree from Purdue University, USA, M.E. degree from Universiti Teknologi Malaysia, Skudai, Malaysia, and $\mathrm{PhD}$ degree from Kanazawa University, Japan, in 2005, 2007 and 2015, respectively. He is currently a faculty member at Universiti Malaysia Pahang. His research interest includes control system design, multi-agent formation control, data-driven based control, and tele-operation. He is a member of IEEE and member of Institution of Engineers (IEM), Malaysia.

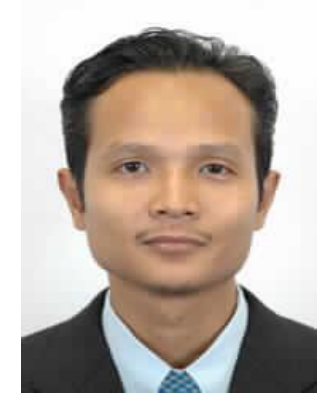

Hamzah Ahmad was awarded B.Sc degree from Shinshu University, Japan, M.Eng degree from UTHM, Malaysia and PhD degree from Kanazawa University in 2000, 2004 and 2011 respectively. He previously works with SREC (M) Sdn Bhd as an engineer and currently with Universiti Malaysia Pahang as an Associate Professor. His research works includes mobile robot navigation, Simultaneous Localization and Mapping, optimal and robust system, artificial intelligence and medical electronics. He is a member of Malaysian Associations of Control Engineers (MACE), IAENG and a Professional Technologist in Malaysian Board of Technologist (MBOT). 